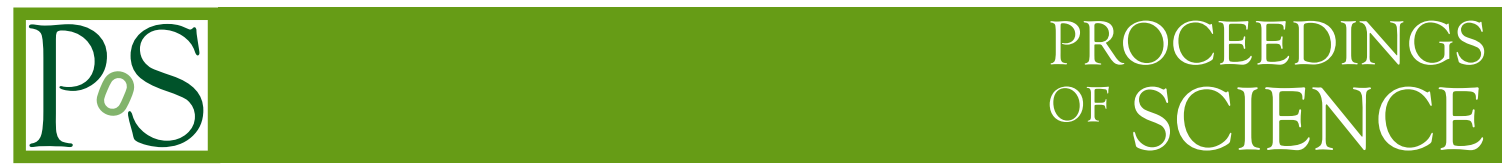

\title{
Hearing the echoes of dark matter and new physics
}

\author{
Fa Peng Huang* \\ Center for Theoretical Physics of the Universe, Institute for Basic Science (IBS), Daejeon 34126, \\ Korea \\ E-mail: huangfpeibs.re.kr
}

Motivated by the absence of dark matter signal in dark matter direct detection experiments and new physics signal at LHC, we study how to hear the echoes of the new physics, especially the dark matter and baryogenesis by new approaches, such as the gravitational wave experiments.

ICHEP2018, XXXIX International Conference on High Energy Physics

4-11 July 2018

Seoul, Korea

${ }^{*}$ Speaker. 


\section{Introduction}

After the discovery of gravitational wave (GW) by aLIGO, GW becomes a new approach to explore the new physics (NP) beyond the standard model and the fundamental problems in particle cosmology. Especially, obvious shortcomings in our understanding of the dark matter (DM) and the baryon asymmetry of the universe (BAU), and no evidence of NP at LHC may give us a hint that a new approach is needed to explore DM and BAU. GW may be used to hear the echoes of DM, baryogenesis, NP models, symmetry breaking patterns of the early universe. We study how to detect DM and NP by GW. Generally, strong first-order phase transition (SFOPT) may be induced in many other NP models, which may produce detectable GW through bubble collisions, turbulence and sound wave mechanisms. There are two typical examples. One is the SFOPT triggered by the $\mathrm{DM}$, the other is the SFOPT in the baryogenesis mechanism.

\section{Probing dark matter and baryogenesis relaxed by phase transition}

In usual asymmetry DM models to explain the DM and BAU, there are usually strong constraints on the DM mass and the reheating temperature. The DM should be about $5 \mathrm{GeV}$, and the reheating temperature should be fine-tuned to avoid washout process. By assuming SFOPT with Q-ball production, the constraints can be significantly relaxed [1]. We start with the following Lagrangian:

$$
\begin{aligned}
\mathscr{L} & =\frac{1}{2}\left(\partial_{\mu} S\right)^{2}-U(S)+\left(\partial_{\mu} \chi\right)^{*}\left(\partial_{\mu} \chi\right)-k_{1}^{2} S^{2} \chi^{*} \chi-\sum_{i} \frac{h_{i}^{2}}{2} S^{2} \phi_{i}^{2} \\
& +\sum_{i} \frac{1}{2}\left(\partial_{\mu} \phi_{i}\right)^{2}-\sum_{a=1,2} \frac{\lambda_{a}^{i j k}}{\Lambda^{2}} \bar{X}_{a} P_{R} D_{i} \bar{U}_{j}^{C} P_{R} D_{k}+\frac{\zeta_{a}}{\Lambda} \bar{X}_{a} Y^{C} \chi \chi^{*}+\text { H.c. }
\end{aligned}
$$

with $U(S)=\lambda_{S}\left(S^{2}-\sigma^{2}\right)^{2} / 4$. In the every early universe, the scalar field $S$ has no vacuum expectation value (VEV) and BAU can be produced by heavy particle decay with the interference effects between two-loop and tree-level diagram. After the BAU is produced, the scalar field $S$ acquires a VEV and SFOPT occurs. With the expansion of the bubbles, the would-be DM particles $\chi$ are packeted into the Q-balls which is guaranteed by the global $U(1)$ symmetry. Q-balls are nontopological solitons. To explain the observed DM and BAU, the final condition can be obtained as

$$
\rho_{D M}^{4} v_{b}^{3 / 4}=73.5\left(2 \eta_{B} s_{0}\right)^{3} \lambda_{S} \sigma^{4} \Gamma^{3 / 4} .
$$

The typical benchmark points are shown in Tab. 1. And the corresponding GW signals are shown

\begin{tabular}{|c|c|c|c|c|c|}
\hline benchmark sets & $\lambda_{S}$ & $e$ & $c$ & $T_{c}[\mathrm{TeV}]$ & $\frac{\sigma}{T_{C}}$ \\
\hline I & 0.008 & 0.754 & 1 & 15.9 & 5 \\
\hline II & 0.0016 & 0.151 & 1 & 6.6 & 5 \\
\hline
\end{tabular}

Table 1: The benchmark sets to satisfy the observed DM density and BAU with $v_{b}=0.3$.

in Fig. 1. This scenario can also have abundant collider phenomenology at LHC. We discuss the collider constraints and predictions at QCD next-leading-order level in Ref. [1]. 

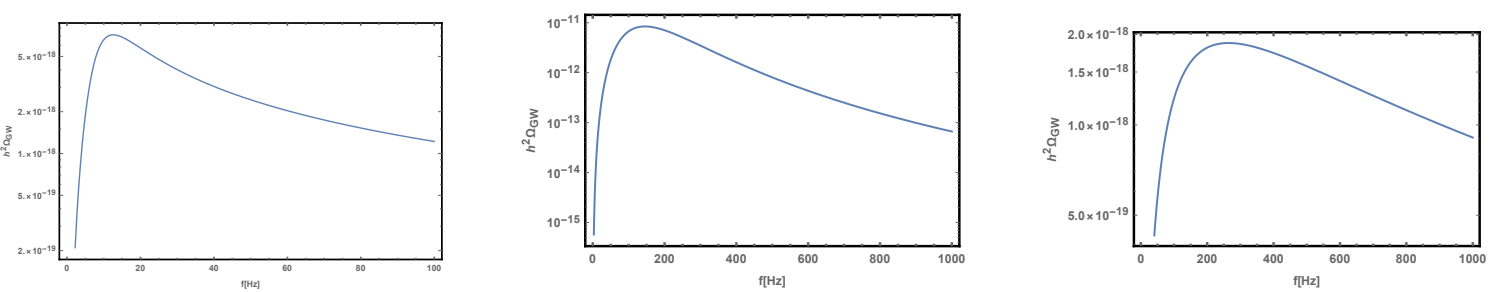

Figure 1: The predicted GW spectrum for benchmark I with $v_{b}=0.3$. From left to right, the line represents the GW spectrum from bubble collision, sound waves and turbulence, respectively.

\section{Exploring the blind spots of dark matter models}

Motivated by the absence of DM signal in DM direct detection (such as the LUX, PandaX-II, XENON1T), a generic classes of scalar DM models have been pushed to the blind spots where dark matter-Higgs coupling is very small. We use the complementary searches via phase transition GW and the future lepton collider signatures to un-blind the blind DM spots. Taking the inert doublet model as an example, we show that GW and CEPC can help to explore the blind spot for this DM model since the SFOPT can still be induced despite the small coupling between the DM matter and the Higgs boson [2]. Considering the DM constraints, the collider constraints and the condition for SFOPT, we show one set of benchmark points $\lambda_{3}=2.84726, \lambda_{4}=\lambda_{5}=-1.41463$ and $M_{D}=59.6 \mathrm{GeV}$ here, where the corresponding DM mass is $64 \mathrm{GeV}$, the pseudo scalar mass and the charged scalar mass are both $299.6 \mathrm{GeV}, \lambda_{h \chi \chi}=\lambda_{345} / 2=0.009$. The corresponding GW signals and collider signals at CEPC are shown in Fig. 2. We also study the GW signals for mixed singlet-doublet model and mixed singlet-triplet model in Ref. [2].

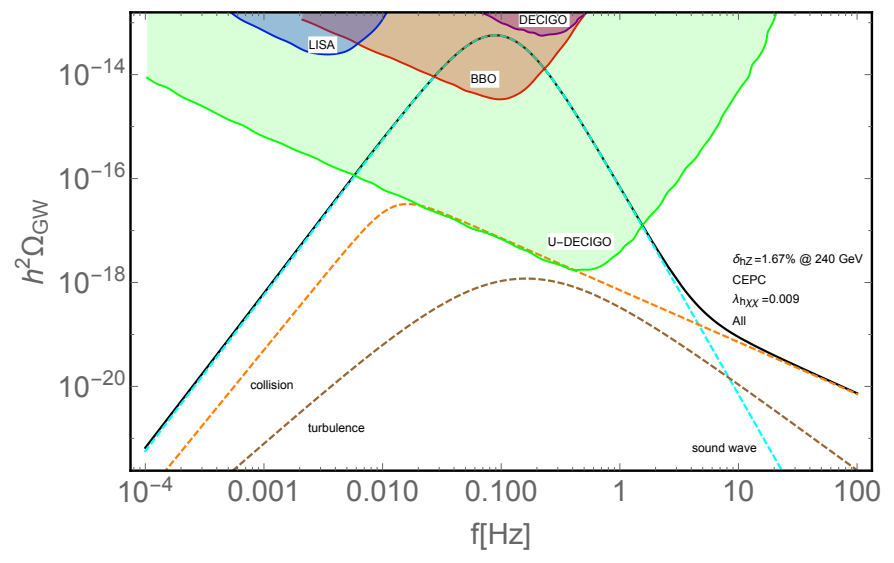

Figure 2: The phase transition GW spectra $h^{2} \Omega_{\mathrm{GW}}$ for the benchmark set in the inert doublet model.

\section{Conclusion and Outlook}

In general, SFOPT can occurs at different energy scale in different NP model from different motivation. The schematic phase transition GW signals are shown in Fig. 3. GW becomes a new 


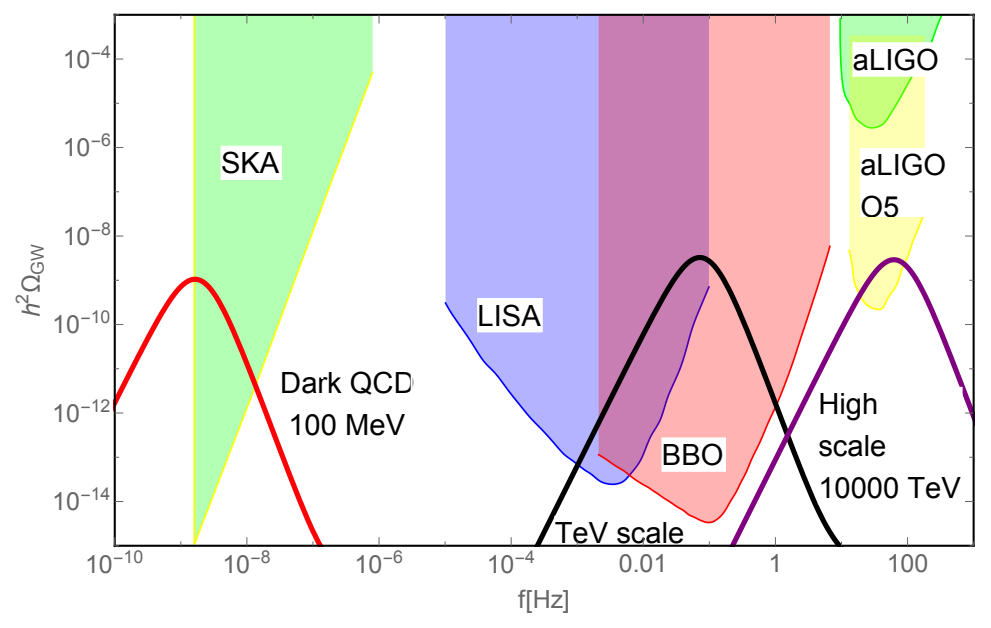

Figure 3: Schematic phase transition GW spectra

and realistic approach to explore DM, baryogenesis and other NP since there are more and more relevant experiments, aLIGO, LISA, SKA, FAST, Tianqin, Taiji)[3, 4, 5, 6, 7].

\section{Acknowlegments}

This work is supported by IBS under the project code IBS-R018-D1.

\section{References}

[1] F. P. Huang and C. S. Li, Phys. Rev. D 96 (2017) no.9, 095028 doi:10.1103/PhysRevD.96.095028 [arXiv:1709.09691 [hep-ph]].

[2] F. P. Huang and J. H. Yu, arXiv:1704.04201 [hep-ph].

[3] F. P. Huang and X. Zhang, arXiv:1701.04338 [hep-ph].

[4] H. Yu, B. M. Gu, F. P. Huang, Y. Q. Wang, X. H. Meng and Y. X. Liu, JCAP 1702 (2017) no.02, 039 doi:10.1088/1475-7516/2017/02/039 [arXiv:1607.03388 [gr-qc]].

[5] F. P. Huang, Y. Wan, D. G. Wang, Y. F. Cai and X. Zhang, Phys. Rev. D 94 (2016) no.4, 041702 doi:10.1103/PhysRevD.94.041702 [arXiv:1601.01640 [hep-ph]].

[6] F. P. Huang, Z. Qian and M. Zhang, Phys. Rev. D 98 (2018) no.1, 015014 doi:10.1103/PhysRevD.98.015014 [arXiv:1804.06813 [hep-ph]].

[7] F. P. Huang, K. Kadota, T. Sekiguchi and H. Tashiro, Phys. Rev. D 97 (2018) no.12, 123001 doi:10.1103/PhysRevD.97.123001 [arXiv:1803.08230 [hep-ph]]. 